\title{
Bimanual Coordination of Bowing and Fingering in Violinists-Effects of Position Changes and String Changes
}

\author{
Oleg Kazennikov and Mario Wiesendanger
}

\begin{abstract}
Music performance is based on demanding motor control with much practice from young age onward. We have chosen to investigate basic bimanual movements played by violin amateurs and professionals. We posed the question whether position and string changes, two frequent mechanisms, may influence the time interval bowing (right)-fingering (left) coordination. The objective was to measure bimanual coordination, i.e., with or without position changes and string changes. The tendency was that the bimanual coordination was statistically only slightly increased or even unchanged but not perceptible. We conclude that the coordination index is limited up to100 ms intervals, without any erroneous perception. Although the mentioned position changes and string changes are movements with their timing, they are executed in parallel rather than in series with the bow-fingering coordination.
\end{abstract}

Keywords: motor control of music, anticipation, serial and parallel functions

In the past, we studied bimanual actions for coordinating the natural handling of tools (Wiesendanger \& Serrien, 2001a, 2001b, 2003). Recently, we have been interested in the motor control of music performance, especially of its bimanual coordination (Baader, Kazennikov, \& Wiesendanger, 2005). Musicians go through a continued training of their fast and complex movements, especially of their hands. Motor control of playing a string instrument is amazingly difficult and needs to be exercised through the entire active musical life. The arm and fingers are the main players of the game, with the underlying postural changes of many body parts. Mastering difficult passages in terms of motor control is only one aspect; the additional decisive musical expression, with its emotional style, identifies the musical artist. Physiological assessment of motor control in music performance offers possibilities to measure various elementary actions. Some aspects of motor control in pianists have already been published by Bernstein \& Popowa (1929) and, more recently, MacKenzie \& Van Eerd (1990) and Soechting, Gordon, \& Engel (1996).

Our first approach was to study some rules of coordination while playing a simple tune on the violin (Baader et al., 2005). The basic coordination is highly

Kazennikov is with the Institute of Problems in Information Transmission, Russian Academy of Science, Moscow, Russia. Wiesendanger is with the Dept. of Medical Sciences, Section of Neurophysiology, University of Fribourg, Fribourg, Switzerland. 
asymmetric, consisting of left-hand fingering on the fingerboard and bowing with the right arm and hand. The bow function is to set the string into vibration with modulations of dynamics and timbre, whereas the left-hand fingers determine intonation, vibrato, and some postural support. One bow stroke may initiate either a new single tone or a tone sequence (e.g., a series of finger stops and finger lifts). In musical terms, each tone is either played détaché (with a separate bow stroke) or legato (a sequence of tones bound under one bow stroke). In a simple tune, played on one string only, we measured the intervals between the finger action and the bow-reversal point, without the complications of position changes along the fingerboard and of string changes. In our first study (Baader et al., 2005), bowfingering intervals could be as long as $100 \mathrm{~ms}$, with means up to $70 \mathrm{~ms}$ in one subject, and 30-40 ms in the five remaining subjects. In all subjects the melody was played without any audible error, even in the subject with the largest deviation of bow-fingering coordination. We therefore concluded that the errors of bow-finger coordination (up to 70-100 ms) may be tolerable in terms of musical feedback. In this context, it is noteworthy to mention the work of Rasch (1979), who measured the auditory synchronization within various ensembles of musicians. Rasch also noted that wind ensembles had shorter timing errors among the players (probably due to their faster rise time of the tones). To cite Rasch for ensemble playing: "a perfect synchronization is not possible in a live performance"; and, "the musicians themselves are in general also unaware of the amount of asynchronization." His best approach was to measure the time difference in professional musicians playing a Beethoven string trio, whereby the asynchrony amounted to 53, 38, 73, and $34 \mathrm{~ms}$ (means of the four trio movements).

The present study deals again with synchronization of bimanual fingering and bowing. In addition, the present approach included also two time-dependent mechanisms: 1) position changes: up or down left-hand shifts along the fingerboard. The tone intervals along the string are nonlinear in comparison with the metric distance of the fingerboard. Series of finger stops, up the string, may include a position change ("jumps" by means of elbow-hand movements) toward a higher or lower position. As an example, a finger stop (tone E on the D-string) may follow by a large position change, i.e., a forearm-hand shift up the fingerboard followed by the next finger stop high up the string. The purpose of position changes is to exploit the whole length of the fingerboard, including the highest tones on the E-string. In terms of motor control, fingering in the highest position is critical because the tones are increasingly more tightly spaced along the whole fingerboard (e.g., Capricci di Paganini). Exploiting a large span of notes on each of the four strings requires persistent practice of the nonlinear spatial fingering up or down on the entire fingerboard. Each position change implies an additional movement. 2) string changes: Each string change requires additional rotational movements of the right bowing arm. For changing to a lower string, a rotation of the bow implies an additional elevation of the elbow whereby the bow is guided from the upper to the lower string. To change from a lower to a higher string the elbow has to adduct. The often frequent bow rotations also require time; rotations around one string are quicker than around three strings. The question is whether the timing of position changes and string changes is working serially or in parallel, or both.

In the current study, the musical scores were more complex (scale, etude) as compared with the previous tune. We hypothesized that the errors between bow 
actions (right) and fingering (left) should be larger than in the previous study (based on a simple tune, without any string changes and position changes). Two additional mechanisms, string changes and position changes, are essential for exploiting the range of violin exercises. Our aim was to investigate the performance of violin players from the motor control perspective, particularly including also the two additional basic mechanisms of string changes and position changes. We now pose the two questions: 1) Do string and position changes affect the temporal bow-fingering coordination? 2) Do the position changes and string changes work in parallel and/or in series? Furthermore, we expected that the professionals synchronized the bimanual bow-finger actions better than the amateurs.

\section{Methods}

\section{Subjects}

Six amateur violinists participated in the study, all having played, as nonprofessionals, also in music ensembles; four professional musicians were also tested with the same criteria.

\section{Recording of Finger and Bowing Trajectories}

We used the Vicon Motion System 460, consisting of four specialized cameras emitting infrared strobes, reflecting back from markers (on left fingers, bow, and violin) to the camera lenses. The Vicon data station controls the cameras and strobes as the incoming signals are collected and digitized. The data are transmitted to a conventional PC containing the Vicon software (data management system), displaying the trajectories from reflecting markers ( $3 \mathrm{~mm}$ diameters) on the four left-hand finger nails (index, middle, ring, and little fingers) and three markers on the bow. Two larger markers (each $1 \mathrm{~cm}$ ) were placed on the violin soundboard and one larger distal marker on the scroll. These three markers defined a triangle of the violin. The sampling rate of the system was set at $200 \mathrm{~Hz}$. In addition, a conventional movie camera (picture and tone) was also used, together with the Vicon video cameras. Static calibration of the workspace and dynamic calibration by waving the wand (stick with two markers) was done to capture the optimal relationship of the marker trajectories; the recognized markers were then automatically labeled for all trials. Each subject was optimally placed in the defined workspace. A model was created, assigning names of the 10 markers (Figure 1A). The markers on the violin were essential because an algorithm automatically transformed the original room coordinates onto violin-centered coordinates to remove the whole-body movements and to correctly obtain only the trajectories of the left-hand fingers and of the bow trajectories controlled by the right-arm movements (detailed description in Baader et al., 2005). Bimanual coordination was measured for each tone as the interval between peak finger velocity and peak of the bow reversal, as shown in Figure 1B.

\section{Tasks}

Each of the two exercises was played five times: (1) tone sequences with and without position changes up and down on one string $=$ pos-task (Ševčik, Violin 


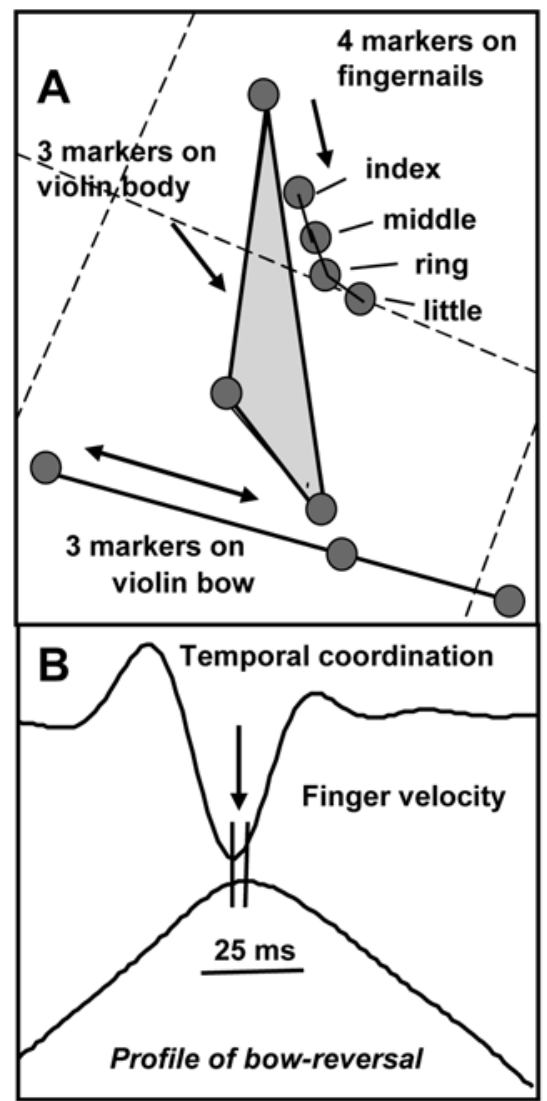

Figure $1-\mathrm{A}$ : The model for creating the four finger trajectories and three bowing trajectories by the Vicon movement recording system. Additional three markers are on the violin, which serve as reference points of the work volume; this eliminates the interfering wholebody movements. B: Measurement of the interval between finger-peak-velocity and peak of bow reversal that is used as the coordination index.

Studies, Opus 8, exercise 29, Bosworth, London); (2) an "artificial" sequence with many string changes, but without position changes $=a$-task.

\section{Data Processing}

Each trial of each subject was inspected by means of the Vicon management software for correct labeling of the individual trajectories. Small gaps in a trajectory were bridged with splines in the Vicon continuity chart; files with (rare) large gaps were discarded. The next step was to import the data (csv-files) into the Matlab application. The measure of interest, i.e., the interval between finger actions (finger stops and finger lifts) and bow reversal, were automatically recorded separately: a) as simple intervals (no position changes or string changes), 
b) as complex intervals with position changes (pos), and c) as complex intervals with string changes (as). A negative interval means that the finger stop occurred before the bow reversal, a positive interval indicates that the finger stop occurred after the bow reversal. The velocity peaks were used as temporal coordination index (CI) to calculate the intervals between finger stops or finger lifts and the bow reversals (Figure 1b).

For each type of exercise a template was created to identify the finger sequence, position changes, and string changes. The results of each subject were short text files providing averages of each exercise, as well as a graphical display of each file. This allowed us to find out whether complex bow-finger intervals significantly differ from simple intervals, thus providing the answer to our main question as detailed above. In ascending sequences, the interval between finger stop and bow reversal was taken as a measure of the coordination index (CI-stop) between the action finger the bow reversal. In descending sequences the coordination index was the interval between finger lift and bow reversal (CI-lift). Intervals CI-stop and CI-lift were analyzed separately. A two-way ANOVA was used for statistical analysis with the subject factor of two independent levels: professionals and amateurs; two levels of dependent factors: with and without position changes or string changes. The level of statistical significance was set at 0.05.

\section{Results}

\section{The Effect of Position Changes on the Coordination Index}

This exercise (pos) consisted of 10 notes, all played on the D-string, with five intervals requiring a position change and five intervals without position changes. Three finger stops were executed up the fingerboard and two finger lifts down (Figure 2). The first position change involved a particularly large up-string position shift, i.e., three full tones and one half-tone. To be in time for the next tone, the index finger has to leave the string in an anticipatory lift to reach the full octave with the little finger. Further shifts are also seen in up position as well as down position changes.

The mean CI between finger stops and bow reversals with position changes was $-35 \pm 27 \mathrm{~ms}$, without position changes $-26 \pm 17 \mathrm{~ms}$ (Table 1). ANOVA did not reveal a difference between amateurs and professionals, $F(1,8)=0.55, p=.48$, nor with position changes vs. no-position changes, $F(1,8)=0.45, p=.52$; there was no interaction between the two factors, $F(1,8)=1.12, p=.32$. The mean CI between finger lifts and bow reversals was $-6 \pm 27 \mathrm{~ms}$ with position change and $29 \pm 15 \mathrm{~ms}$ without position change. ANOVA showed a significant difference of the CI-lift with and without position changes, $F(1,8)=10.51, p<.05$. The difference of the CI-lift between professionals and amateurs was not significant, $F(1,8)$ $=0.01, p=.93$, as well as the interaction factor, $F(1,8)=2.17, p=.17$.

\section{The Effect of String Changes on the Coordination (As-Task)}

Figure 3 illustrates the exercise with many string changes consisting of 26 notes requiring 16 string changes (nine finger stops and seven finger lifts) and 10 notes without string changes (five finger stops and five finger lifts). 


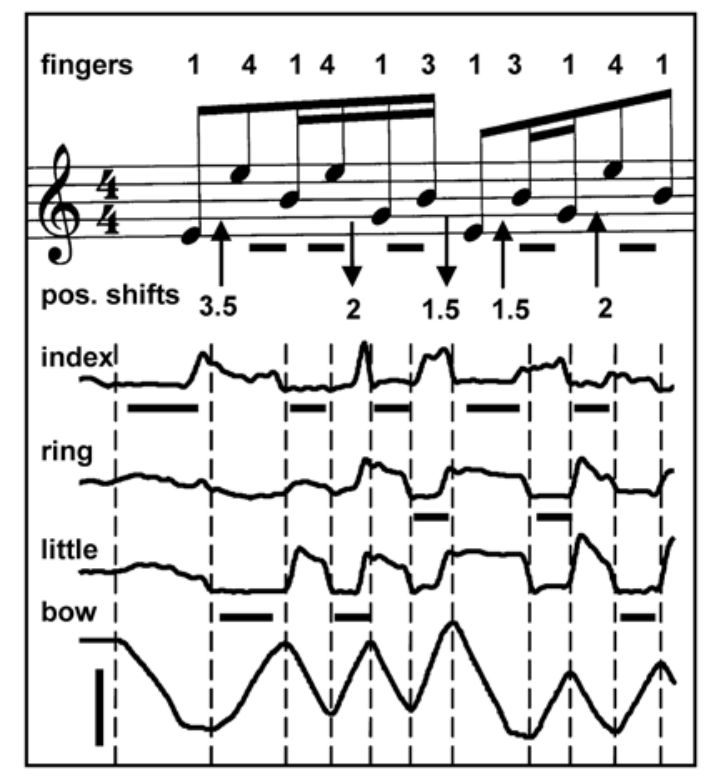

Figure 2 - Short exercise pos (from Ševčik, Violin Studies, Opus 8, exercise 29, Bosworth, London) was played and repeated five times by each subject. Uppermost line: sequence of finger actions ( $1=$ index, $2=$ middle, $3=$ ring, $4=$ little fingers $)$. Under the notation bar there are position shifts (three arrows up and two arrows down; the position shifts vary from 1.5 tones to 3.5 tones). The horizontal bars indicate no position shifts. Below are displacement profiles of the four fingers. Finger stops are indicated by horizontal flat portions. The vertical dashed lines indicate the timing of the bow reversals. Note that the first index finger leaves the fingerboard slightly before the tone (see also flat portion of the corresponding bow action) because the large whole arm position change needs its time. This does not, however, disturb the flow of music because the string vibrations do not stop immediately.

Without string change, the finger pressed the string earlier $(-29 \pm 9 \mathrm{~ms})$ than the finger stops with string changes $(-41 \pm 13 \mathrm{~ms})$. This difference was significant, $F(1,8)=19.34, p<.05$, but no difference was observed between professionals and amateurs, $F(1,8)=0.62, p=.45$. There was also no interaction factor, $F(1,8)=$ $0.06, p=.81$. Without string changes the finger lift intervals were $51 \pm 21 \mathrm{~ms}$, with string change $45 \pm 17 \mathrm{~ms}$. Finger-lift intervals differed insignificantly with and without string changes, $F(1,8)=1.55, p=.25$. Professional and amateur groups did not differ, $F(1,8)=2.71, p=.14$, and no interaction was observed, $F(1,8)=$ $0.07, p=.80$; see also Table 1 .

In Figure 4, we illustrate single examples of a position change along the fingerboard of the violin and an example of a string change with rotational movements for reaching a higher or lower string. Both mechanisms are additional movement categories. In Figure 4A, a position change that is particularly large corresponds musically to an octave between the index finger (tone E on D-string) and reaching with a whole-hand shift along the fingerboard, with the little finger 


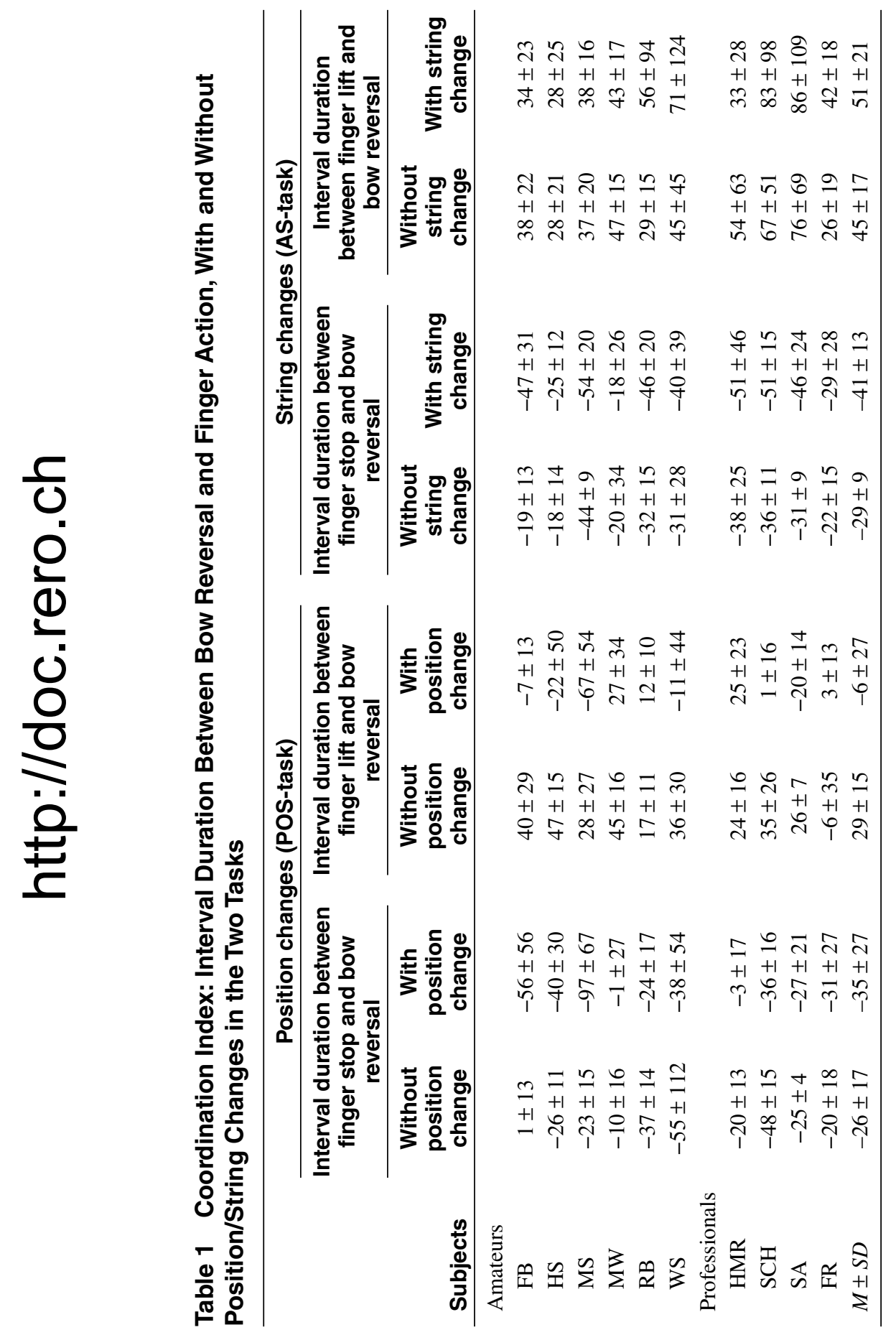




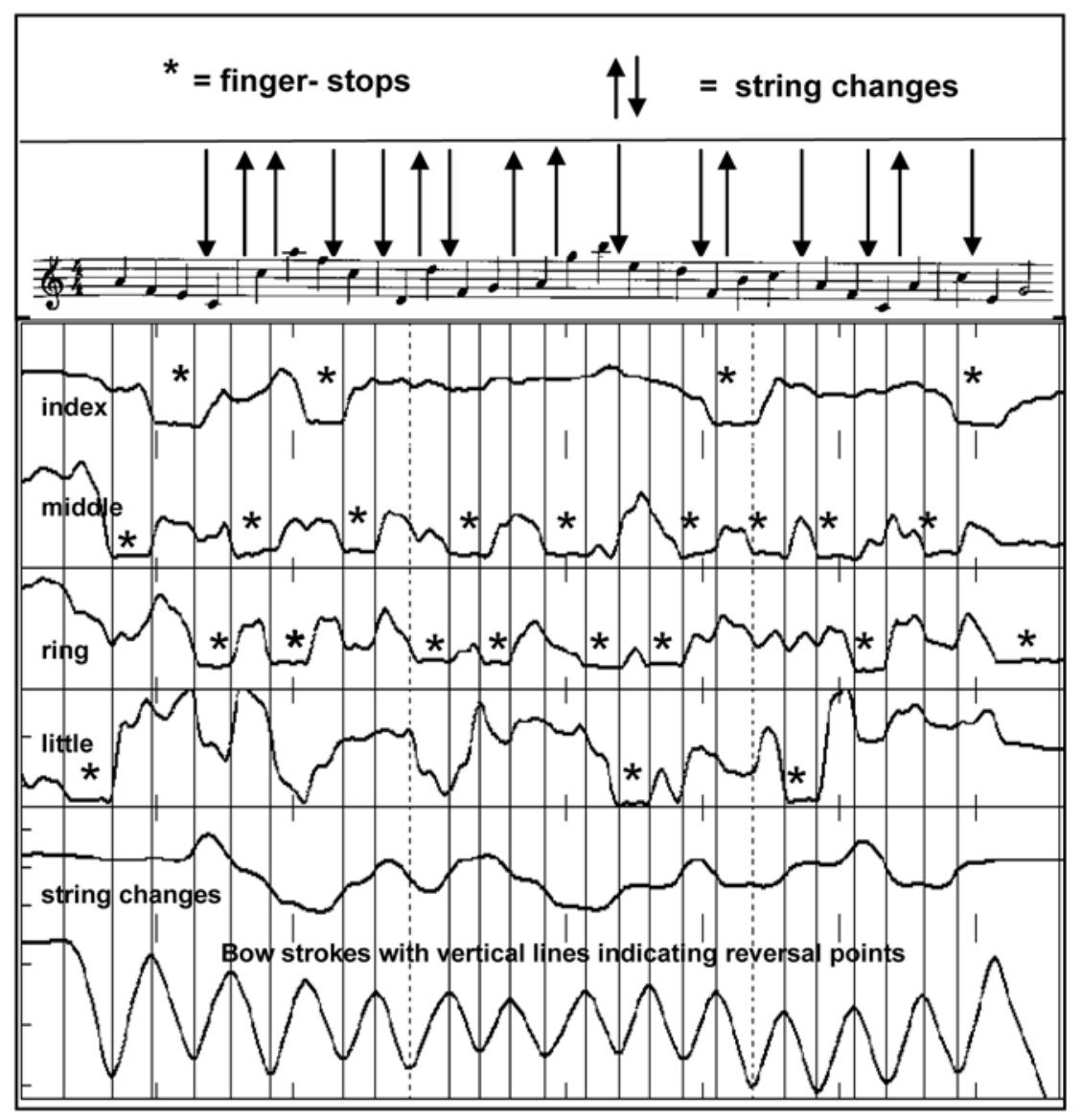

Figure 3 - Exercise of many string changes, but without position changes. Arrows (up and down) signal the bow changes to upper or lower strings. Finger stops are indicated with the flat portion and an asterisk. The vertical lines indicate the reversal points of the bow actions at the bottom. Above is the curve with kinks indicating the occurrence of string changes.

stretching out for the finger stop on the same string. The whole-hand shift amounts to a span of 3.5 tones. In Figure 4B, one can see finger stops changing from the E-string to the two lower D- and G-strings.

The primary straight bow movements are parallel to the transverse bridge. But string changes involve movements to a higher or lower string for executing appropriate finger stops involving bow rotations. The changes of few degrees of the bow angle have to be highly precise for the step-wise selection of the proper string. These angular movements function in parallel to the straight bow movements. Figure 5 illustrates these angular changes, which must be extremely precise, depending whether string changes include one, two, or three strings. Amazingly, the fine motor control depends on precise angles executed to a large 


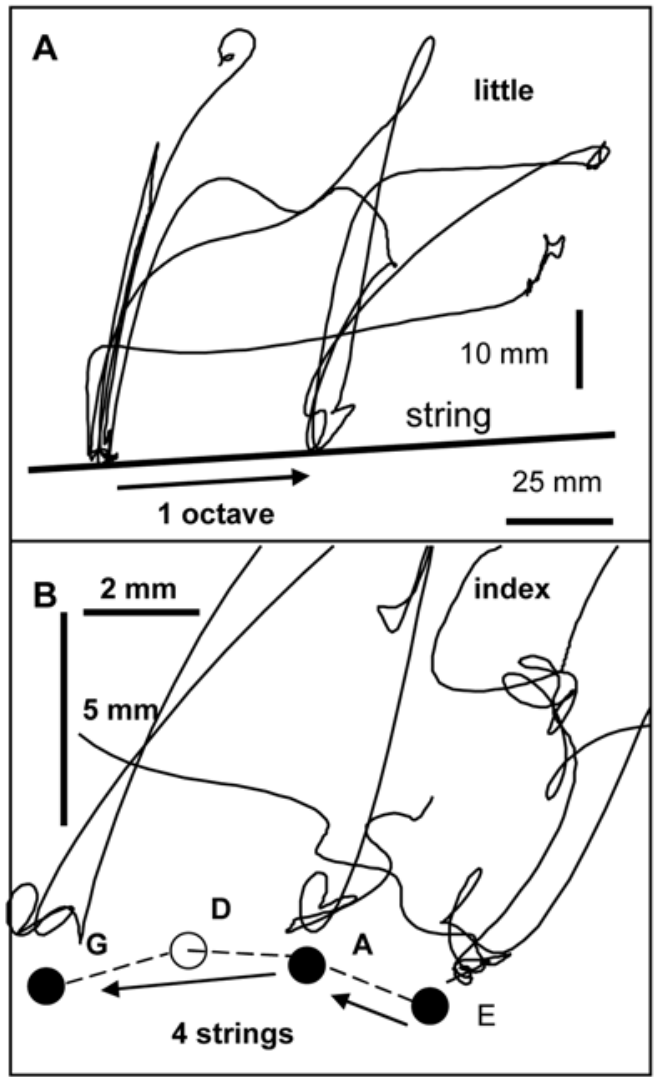

Figure $4-$ A: Trajectory of the little in the vertical plane parallel to the string. Example of three finger stops of the little, followed by a large position change, i.e., a shift of the whole hand along the string. Landed higher up on the string, the same finger makes two more finger stops. B: Trajectory of the index in the vertical plane transverse to the string. The index finger moves transversely, i.e., from the E-string to the A- and G-strings (marked black). The three finger stops require two sequential string changes (arrows) without touching the D-string.

part by the proximal shoulder adductors (to a higher string) or elevators (to a lower string).

Figure 6A illustrates the three principal mechanisms of fingering on the fingerboard. On the left (filled circle) denotes an actual finger stop, e.g., of the middle finger, which is followed by a next stop of the little finger higher up on the string. This action ends the lower tone and initiates the upper tone-a purely serial sequence. The middle (filled) finger is followed by an index finger stop below. The timing of the lower tone is, however, determined by a lift of the upper finger. In addition, the lower finger may anticipate the finger stop as long as the upper finger is still on the string. Anticipation is a very important mechanism that can also function when a tone sequence involves a string change, either down-string or 
up-string. This is illustrated on the right side. Again the initiation of a new tone is dependant on the bow string change where the finger may already be placed. In Figure 6B (left) one can see an original serial sequence typical for an ascending step; on the right the anticipation (arrow) is typical for a descending step on the same string or when string changes occur.

\section{Discussion}

The relatively complex movement categories of the violinist indicate that music performance, similar to language, is composed of a multitude of skillful movements that run in series as well as in parallel. Fingering of the left hand along the extension of the fingerboard is crucial but radically different from an apparatus of an electronic key-press gadget. Musical sequences, played on the violin, need correct timing and intonation (pitch); tone onset and tone termination typically involve two finger actions.

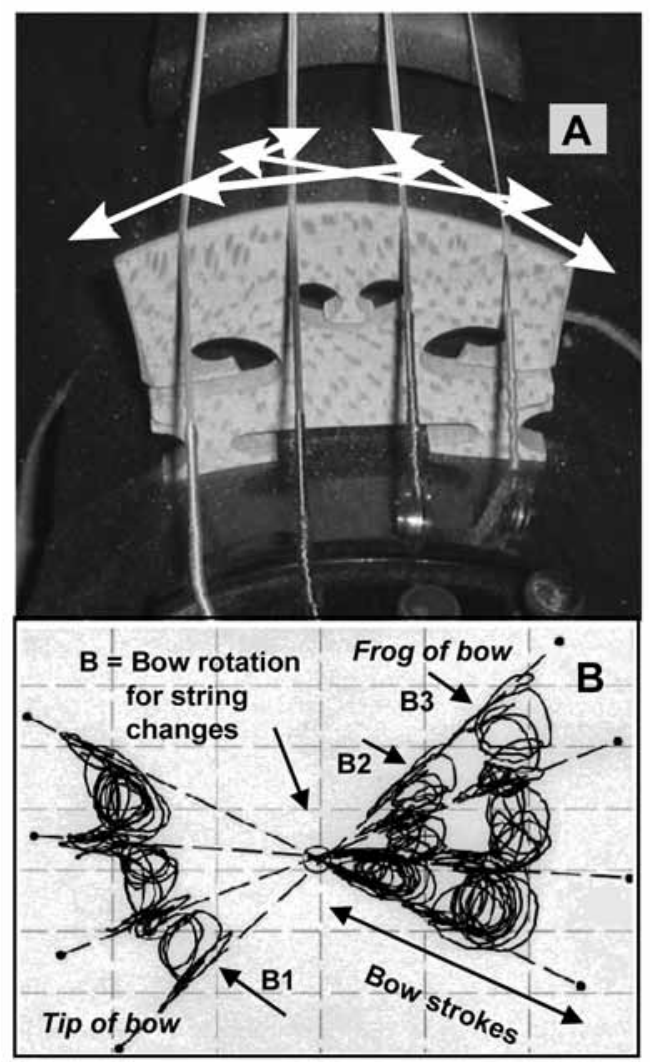

Figure 5-A: Visualization of the rectilinear bow strokes and the angular movements for string changes. B: Front view of rectilinear bow strokes and simultaneous angular movements of the bow (indicated by two arrows) during frequent string changes. 
In the current study, we focused on position changes and string changes and asked whether these mechanisms influence the principal bow-fingering coordination. We observed some statistically valid prolongation effects of position-change effects and string-change effects. These were observed in amateurs as well as in professionals. This outcome was puzzling because we could not statistically distinguish from the two groups. As has been previously discussed, Rasch (1979) was probably first to discover the deviations from zero ms synchronizations in string players (which is less the case for playing other instruments). Up to $100 \mathrm{~ms}$ deviations had been noted in his data, and now also in our own data, including in good players. It appears therefore that our results are

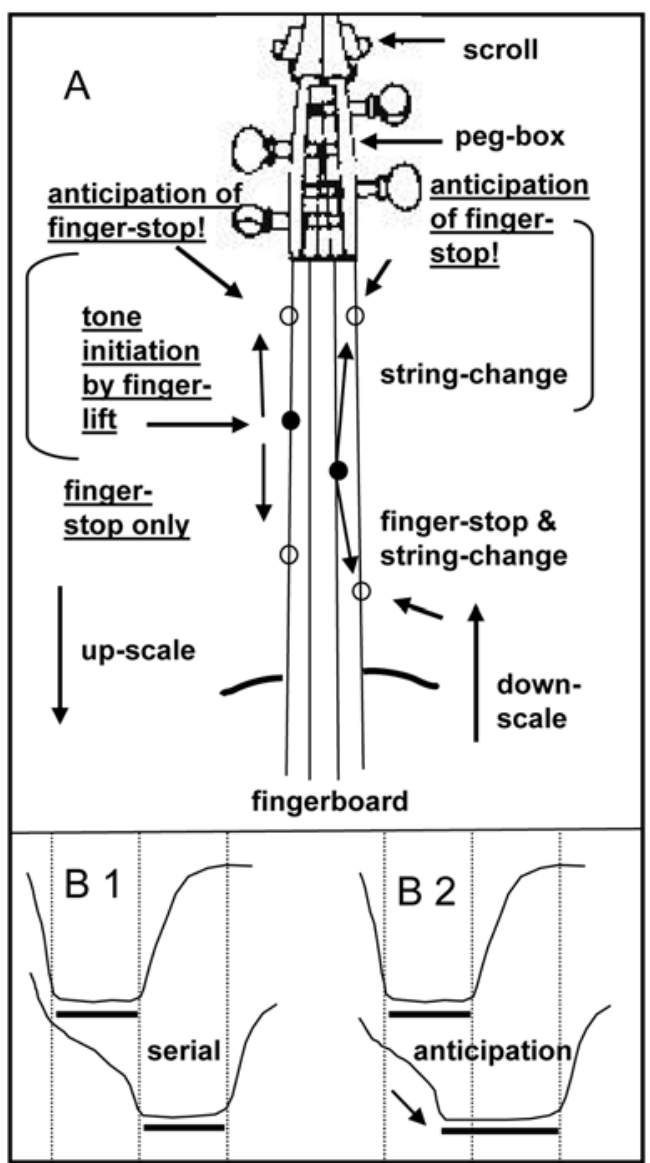

Figure 6 - A: Up-scale from black finger stop to a finger stop (white): a pure serial action as shown in B1. Down-scale from black finger stop to white finger stop: upper finger must be lifted to initiate lower tone and the finger stop below must be already present, i.e., anticipation as also shown in B2. On the right: string changes allow for anticipation in both directions, corresponding to $\mathrm{B} 2$. 
compatible with the measurements of Rasch (1979), although the present measurements were obtained in different situations. His best approach was to measure the time difference between professional players of a string trio whereby values of $50 \mathrm{~ms}$ deviation from zero were observed. Our approach was to test the bimanual coordination index, i.e., the difference between the right bow-reversal point and the finger stop. The hypothesis was that additional and frequent occurrences of string changes and position changes would reveal longer timing of the bow-fingering interval. This was partly the case, but now appears to be statistically nonrelevant. Another conclusion is that the string changes and position changes are also time dependent, but the above changes are likely to happen in parallel, possibly only little in series. Anticipatory finger actions are frequent and important; typically, movements run serially as well as in parallel.

All musicians have to learn over years to master the instrument with the assistance of a teacher. There is a massive literature of scores, especially etudes, perhaps similar to learning a new language.

The linguist studies the rules and mechanisms of how sentences are created. The conductor Leonard Bernstein was interested in some similarities in music and language. In his book he wrote chapters on "musical syntax" and "musical semantics" (Bernstein, 1976). The language is structured in words and sentences. According to The new Encyclopedic Dictionary of the English Language (1971), "a syllable is the least expression of language or thought, not of a single character." Similarly, in reading for playing music, the eyes capture chunks of notes (sight-reading) in anticipation, not single notes, followed by the motor control (Land, 2006). Reading and playing music thus consists also of serial and a lot of parallel processing. The string instrument is based on fingering at the right time prescription, with a correct intonation, as well as rendering the tune by means of the right-hand bow strokes. To play short passages of music implies also to master biomechanical rules, of course primarily both hands and arms but also wholebody posture for support. The participants were asked to play excerpts of etudes containing intervals with string changes or position changes. In the etudes there were also intervals without string changes or position changes. Our goal was to test whether string changes and/or position changes influence the timing in bimanual bow-finger coordination.

Above, we shortly mentioned another crucial and basic mechanism-correct intonation. In view of the difficult methodology of measuring pitch in our experimental situation, we abstained to further complicate the present task. We are aware that the ingredients of music, like a violin solo sonata of Johann Sebastian Bach, cannot be compared with the simple etudes we have selected. Our aim is to investigate some building blocks containing the particular motor mechanisms of position and string changes that must be learned early in music education

\section{Acknowledgments}

We are grateful to Professor Eric Rouiller, Head of Neurophysiology at the University of Fribourg, Switzerland, who offered us a laboratory for investigating motor control in violin playing. 


\section{References}

Baader, A.P., Kazennikov, O., \& Wiesendanger, M. (2005). Coordination of bowing and fingering in violin playing. Cognitive Brain Research, 23, 436-443.

Bernstein, L. (1976). The unanswered question - six talks at Harvard. Cambridge, MA: Harvard University Press.

Bernstein, N., \& Popowa, T. (1929). Untersuchung über die Biodynamik des Klavieranschlags. Arbeitsphysiologie, 1, 396-432.

Land, M.F. (2006). Eye movements and the control of actions in everyday life. Progress in Retinal and Eye Research, 25, 296-324.

MacKenzie, C.L., \& Van Eerd, D.L. (1990). Rhythmic precision in the performance of piano scales: Motor psychophysics and motor programming. In M. Jeannerod (Ed.), Attention and performance XIII, motor representation and control (pp. 375-408). Hillsdale, NJ: L.Erlbaum Ass.

Rasch, R.A. (1979). Synchronization in performed ensemble music. Acustica, 43, 121131.

Soechting, J.F., Gordon, A.M., \& Engel, K.C. (1996). Sequential hand and finger movements: Typing and piano playing. In J.R. Bloedel, T.J. Ebner, \& S.P. Wise (Eds.), The acquisition of motor behavior in vertebrates (pp. 343-360). Cambridge, MA: MIT Press.

Wiesendanger, M., \& Serrien, D.J. (2001a). Neurological problems affecting hand dexterity. Brain Research Reviews, 36, 161-168.

Wiesendanger, M., \& Serrien, D.J. (2001b). Toward a physiological understanding of human dexterity. News in Physiological Sciences, 16, 228-233.

Wiesendanger, M., \& Serrien, D.J. (2003). The quest to understand bimanual coordination. In S. Mori, D.G. Stuart, \& M. Wiesendanger (Eds.), Brain mechanisms for the integration of posture and movement (pp. 491-505). Amsterdam: Elsevier. 